\title{
Estresse ocupacional em enfermeiros intensivistas que prestam cuidados diretos ao paciente crítico
}

\author{
Occupational stress in intensive care nurses who provide direct care to critical patients
}

Estrés laboral de enfermeros intensivistas que prestan atención directa al paciente crítico

\section{Kelly Cristina Inoue', Gelena Lucinéia Gomes da Silva Versa", Ana Cláudia Yassuko Murassaki"', Willian Augusto de Meloiv, Laura Misue Matsudav}

' Universidade Estadual de Maringá, Hospital Universitário Regional de Maringá. Maringá-PR, Brasil. " Universidade Estadual do Oeste do Paraná, Hospital Universitário do Oeste do Paraná. Cascavel-PR, Brasil.

III Santa Casa de Misericórdia de Maringá. Maringá-PR, Brasil.

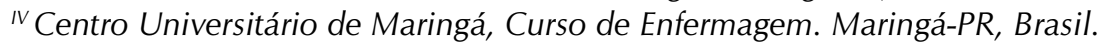

$\checkmark$ Universidade Estadual de Maringá, Departamento de Enfermagem. Maringá-PR, Brasil.

\section{Submissão: 07-04-2012 Aprovação: 04-09-2013}

\section{RESUMO}

Realizou-se pesquisa descritivo-exploratória em cinco instituições hospitalares da região Oeste do estado do Paraná, entre maio e julho de 2010, com o objetivo de identificar o nível de estresse em enfermeiros intensivistas que prestam cuidados diretos a pacientes críticos. Participaram 58 enfermeiros intensivistas assistenciais, cujo nível de estresse foi avaliado de acordo com o Domínio D da Escala Bianchi de Estresse. A maioria (65,5\%) apresentou nível de estresse médio. Os estressores com maior pontuação foram Enfrentar a morte (5,6 pontos); Atender às emergências na unidade (5,1 pontos); Atender os familiares de pacientes críticos ou Orientar familiares de pacientes críticos (ambos com 4,8 pontos). É preciso adotar estratégias para a prevenção / redução de estresse, porque os principais estressores apontados relacionam-se a atribuições específicas dos enfermeiros.

Descritores: Assistência de Enfermagem; Enfermeiros; Estresse Psicológico; Unidades de Terapia Intensiva.

\begin{abstract}
In order to identify the stress level of nurses that provide direct care to critically ill patients, it was carried out a descriptive and exploratory study in five hospitals of the western region of the state of Paraná, Brazil, from May 2010 to July 2010. Fifty-eight intensive nurses participated in the study, whose stress level was evaluated according to the Domain D of Bianchi's Stress Scale. Most $(65.5 \%)$ of them had medium stress levels, The stressors that obtained the highest levels were: facing death (5.6 points); answering emergency calls unit (5.1 points); caring for critically ill patients' or talking to family members of critically ill patients (both 4.8 points). It is needed to adopt stress prevention and reduction strategies to its minimization or elimination, because the main stressors mentioned are directly related to the duties of nurses.
\end{abstract}

Key words: Nursing Care; Nurses; Stress; Psychological; Intensive Care Units.

\section{RESUMEN}

Con el fin de identificar el nivel de estrés de enfermeros que prestan atención directa a pacientes críticamente enfermos, se realizó un estudio descriptivo y exploratorio, en cinco hospitales de la región oeste del estado de Paraná, Brasil, de mayo a julio de 2010. Participaron del estudio 58 enfermeros de cuidados intensivos, cuyo nivel de estrés se evaluó de acuerdo con la categoría D de la Escala de Estrés de Bianchi. La mayoría (65,5\%) tenía un nivel medio de estrés. Los factores de estrés con más alta puntuación fueran: Enfrentar la muerte (5,6 puntos); Asistir a las emergencias en la unidad (5,1 puntos); Proporcionar atención a familiares de pacientes críticamente enfermos o Orientar familiares de pacientes críticamente enfermos (ambos 4,8 puntos). Es necesario adoptar estrategias para la prevención / reducción del estrés, ya que los principales factores de estrés se relacionan a deberes específicos de los enfermeros.

Palabras clave: Atención de Enfermería; Nurses; Estrés Psicológico; Unidades de Cuidados Intensivos. 


\section{INTRODUÇÃO}

O avanço tecnológico e científico, que tem produzido meIhorias nas condições de vida e de saúde da população, tem também provocado doenças laborais, muitas vezes causadas pela elevada carga de trabalho e incorporação tecnológica nos sistemas de produção.

A busca da produtividade a qualquer custo esbarrou nos limites do próprio ser humano e resultou no aumento do seu sofrimento. Desse modo, houve ambiente propício ao desenvolvimento da Teoria do Estresse, que surgiu no contexto da explosão da produção e do consumo e aborda sobre fatores desencadeantes do adoecimento físico e psicológico ${ }^{(1-2)}$.

Diante da evolução e da globalização tecnológica, os trabalhadores precisam lidar com o aumento da demanda de aprendizagem de novas habilidades; adaptação a diferentes formas de trabalho; exigências cada vez maiores à alta produtividade e máxima qualidade dos produtos/serviços em tempo reduzido; maior competitividade no mercado de trabalho; condições laborais precárias; menores benefícios empregatícios; além do menor tempo para o convívio social ${ }^{(3)}$. Num contexto assim, o ambiente de trabalho pode ser responsável pelo desenvolvimento de muitas doenças, como por exemplo, o estresse ocupacional.

O termo estresse tem sido utilizado para designar sensações desagradáveis e/ou de desconforto diante às diferentes situações percebidas pelo próprio indivíduo que, se utiliza de seu processo psicológico e de sua compreensão para julgar os fatos, concebendo-os como estressantes ${ }^{(4)}$.

Ao considerar que a interação de condições variadas propicia ambientes de trabalho mais ou menos favoráveis à saúde ${ }^{(5)}$, o labor pode ser fonte de estressores que condicionam ao estresse ocupacional, que ocorre entre os profissionais incapazes de enfrentar as demandas requeridas pela sua ocupação.

O estresse ocupacional, entretanto, não é um fenômeno novo, mas sim um novo campo de estudo que ganhou destaque em razão do aparecimento de doenças que a ele foram vinculadas, tais como a hipertensão, a úlcera, entre outras ${ }^{(1)}$.

No contexto da enfermagem, os enfermeiros quase sempre atuam em meio a riscos e condições desfavoráveis que podem influenciar diretamente na sua saúde física e mental, resultando em estresse e prejuízos ao trabalho(6). Essa condição ocorre porque as atividades conferidas legalmente ao enfermeiro demandam muita atenção, discernimento e responsabilidade, fazendo com que os fatores psicossociais desencadeados pelas atividades laborais desse profissional condicionem o aparecimento do estresse no trabalho ${ }^{(7)}$.

Reconhece-se que, no ambiente hospitalar, devido ao convívio diário com a gravidade da doença, o sofrimento humano e a morte, a enfermagem é considerada como profissão altamente estressante, se comparada às outras profissões da área da saúde ${ }^{(8)}$ e isso é mais evidente em setores críticos como a Unidade de Terapia Intensiva (UTI) ${ }^{(9)}$ em que a expectativa constante de descompensação dos pacientes graves, associada à complexidade assistencial, inerente à concentração tecnológica avançada, torna o ambiente e o trabalho nesse Serviço, mais difícil e desgastante ${ }^{(10)}$.
A literatura aponta que a UTI representa uma área de atuação particularmente estressante para a enfermagem ${ }^{(11-12)}$, em razão de múltiplos fatores como a alta mortalidade dos pacientes, o que, mediante a sua ocorrência, gera tensão e ansiedade nos profissionais que se questionam sobre o seu próprio empenho e qualidade da assistência prestada ${ }^{(11)}$.

Em estudo realizado com enfermeiros de UTI de cinco hospitais do Estado de São Paulo, foi verificado que 57,1\% avaliaram sua unidade de trabalho como fonte geradora de estresse e, apenas $9,6 \%$ não percebiam a UTI como um local estressante ${ }^{(4)}$.

As representações sociais referentes ao trabalho, elaboradas por enfermeiras de cinco UTI de dois hospitais de grande porte de Salvador, Bahia, evidenciaram as palavras estressante, responsabilidade, assistência integral e gratificante, como elementos do núcleo central. Essa miscelânea de evocações livres de palavras impede que os gestores se preocupem com intervenções eficazes para a redução do estresse laboral, uma vez que a assistência integral também é entendida como gratificante $^{(10)}$.

Para que os gestores atuem em favor da minimização do estresse na enfermagem, em especial no enfermeiro que atua em UTI, é importante que se detecte e atue sobre os fatores causadores desse fenômeno, com vistas ao planejamento e implementação de estratégias que contribuam para a adequação do ambiente ao desempenho do trabalho.

Um instrumento que possibilita a análise da variação do nível de estresse de enfermeiros, em qualquer tipo de unidade de atuação, é a Escala Bianchi de Stress (EBS), composta por seis domínios: (A) Relacionamento com outras unidades e supervisores; (B) Funcionamento da unidade; (C) Administração de pessoal; (D) Assistência prestada ao paciente; (E) Coordenação das atividades; e, (F) Condições de trabalho ${ }^{(13)}$.

Dentre os domínios citados, o domínio $\mathrm{D}$, que se refere à Assistência prestada ao paciente ${ }^{(13)}$, será o foco deste estudo porque a assistência direta ao paciente grave e a realização de cuidados diretos de alta complexidade, tão evidentes em UTI, são de responsabilidade privativa do enfermeiro, conforme consta no Decreto-Lei ${ }^{\circ}$. 94.406/87 que regulamenta o Exercício Profissional da Enfermagem ${ }^{(14)}$.

No intuito de levantar informações acerca do estresse em enfermeiros questiona-se: Como se apresenta o nível de estresse em enfermeiros que prestam cuidados diretos ao paciente crítico? E para responder a essa questão se propõe a realização do presente estudo cujo objetivo consiste em identificar o nível de estresse apontado por enfermeiros intensivistas que prestam cuidados diretos aos pacientes críticos.

\section{MÉTODOS}

Estudo analítico, transversal e quantitativo, realizado entre maio e julho de 2010 nas UTI Adulto, Pediátrica e Neonatal, de cinco instituições hospitalares da região Oeste do estado do Paraná, as quais foram denominadas aleatoriamente de A, B, C, D e E.

A instituição A é um hospital de ensino público, possui UTI Adulto, Neonatal e Pediátrica, cada qual composta por 
nove, cinco e 10 leitos, respectivamente. Nesse hospital os profissionais de enfermagem trabalham 40 horas semanais e, no período noturno, atuam sob o regime de 12 horas de trabalho por 60 horas de descanso.

Tanto na UTI Adulto quanto na UTI Neonatal da instituição A existem em média cinco Técnicos de Enfermagem (TE) e um Enfermeiro exclusivo para o setor por turno; enquanto na UTI Pediátrica atuam dois TE e um Enfermeiro. Destaca-se que, além dos enfermeiros que assistem diretamente o paciente e supervisionam sua respectiva equipe de trabalho, há ainda, um enfermeiro administrativo para cada uma destas UTI.

Sobre as instituições B, C, D e E, cabe mencionar que essas são instituições privadas e totalizam 46 leitos de UTI Adulto, de diversas clínicas, em especial a oncologia na instituição C e a cardiologia nas instituições D e E.

Quanto ao número de leitos para a UTI Adulto, as instituições B e C dispõem cada uma de 10 leitos; a instituição D possui 11 leitos; a instituição E tem 15 leitos, dos quais cinco são destinados à especialidade de cardiologia. É importante ressaltar ainda que, na instituição $E$, existem também 11 leitos de UTI Neonatal.

Em cada um dos hospitais privados, a equipe de enfermagem trabalha 40 horas semanais, mas, diferentemente da instituição $\mathrm{A}$, a equipe do período noturno atua sob o regime de 12 horas de trabalho por 36 horas de descanso.

Ressalta-se que cada uma das UTI das instituições (B, C, $\mathrm{D}$ e E) conta com uma média de quatro TE e um enfermeiro assistencial em cada turno de trabalho e esse enfermeiro (que participou deste estudo), por não haver um profissional específico para exercer o papel de gerente / coordenador do setor, é quem desempenha concomitantemente as atividades gerenciais de seus respectivos turnos.

Para seleção dos sujeitos da pesquisa, consideraram-se como critérios de inclusão: ser enfermeiro(a) intensivista com mais de três meses em exercício na função no Serviço investigado, e aceitar formalmente participar do estudo. Foram excluídos da pesquisa, aqueles que desempenhavam exclusivamente atividades de gerenciamento e coordenação no âmbito setorial $(n=2)$. Com isso, de um total de 62 enfermeiros, a população do estudo foi composta, por 58 enfermeiros assistenciais.

Quanto aos aspectos éticos e legais vigentes, todos os quesitos foram cumpridos e essa pesquisa encontra-se registrada no Comitê Permanente de Ética em Pesquisas com Seres Humanos (COPEP) da Universidade Estadual de Maringá (UEM), sob o Parecer $n^{\circ} 421 / 2010$.

Para a coleta de dados, utilizou-se a EBS que consiste em uma escala do tipo Likert, de auto-preenchimento, cujas respostas variam de 1 (Pouco Desgastante) a 7 (Altamente Desgastante); sendo que a pontuação 4 consiste em valor neutro e o zero se refere à não realização da atividade avaliada ${ }^{(13)}$.

A EBS é constituída por dados de caracterização sociodemográfica (Parte 1) e 51 itens (Parte 2) referentes às atividades realizadas pelo enfermeiro e/ou condições de trabalho, denominadas de estressores. Entretanto, para fins desta pesquisa, foram consideradas apenas as questões referentes à Assistência prestada ao paciente (Domínio D), formada por 15 itens, do 16 ao $30^{(13)}$.
Os dados foram compilados e tratados em banco de dados eletrônicos, cuja análise estatística descritiva e inferencial ocorreu mediante o uso do programa SPSS 20. Para a estatística descritiva, foram calculadas as frequências, as porcentagens, a variação mínima e a máxima, a média e o desvio padrão (DP), tanto das variáveis que compõem a Parte 1 quanto daquelas que compõem a Parte 2 do instrumento de coleta de dados.

A análise dos dados da Parte 2, qualificação do nível de estresse global, foi realizada de acordo com a categorização de Bianchi, a qual preconiza que um domínio considera as atividades ou características pertinentes ao trabalho (estressores) e a sua média, representa o nível de estresse, classificado como: Baixo nível se o escore for igual ou abaixo de 3,0 pontos; Médio nível se o escore se situar entre 3,1 e 5,9 pontos; e Alto Nível se o escore for igual ou superior a 6,0 pontos ${ }^{(13)}$.

O escore médio do Domínio D da EBS foi utilizado ainda para testar associação do nível de estresse com variáveis sociodemográficas e profissionais, considerando-se nível de significância de $5 \%$. As variáveis com $p$-valor $<0,20$ na análise univaridada foram incluídas na regressão linear multivariada, para ajuste do modelo.

\section{RESULTADOS}

A população de estudo foi composta por 58 enfermeiros assistenciais, cuja idade variou de 23 a 43 anos, com média de $31 \pm 5,2$ anos de idade; $53(91,4 \%)$ deles com menos de 40 anos. Desses, 39 (68,4\%) eram mulheres, 38 (65,5\%) eram casados, $34(60,7 \%)$ eram chefes de família e 28 (49,1\%) possuíam filhos.

Com relação à formação profissional, 32 (55,2\%) eram especialistas e a maior titulação foi a de mestre (3,4\%); com até 15 anos de graduado em Enfermagem. Ressalta-se que 24 $(41,4 \%)$ trabalhavam em instituição pública e 34 (58,6\%) em instituição privada; 36 (63,2\%) em UTI Adulto e 21 (36,8\%) em UTI Infantil; com até 10 anos de atuação em UTI.

Apesar de atuarem como enfermeiros assistenciais, 5 $(8,6 \%)$ apontaram desempenhar também a função gerencial; a jornada semanal de trabalho era de 30 a 56 horas. Nesse contexto, 29 (50,9\%) enfermeiros cumpriam carga horária de 36 horas semanais e outros $24(42,1 \%)$, de 40 horas semanais; 44 (75,9\%) possuíam vínculo empregatício único e $43(74,1 \%)$ recebiam renda mensal entre três e 10 salários mínimos. Destaca-se que $24(41,4 \%)$ dos entrevistados se sentiam satisfeitos com a remuneração, seguidos de 18 (31\%) que apontaram estar pouco satisfeitos, 8 (13,8\%) pouco insatisfeitos e $4(6,9 \%)$, respectivamente, referiram estar muito satisfeitos ou muito insatisfeitos.

No tocante ao nível de estresse relacionado à Assistência prestada ao paciente, 38 (65,5\%) enfermeiros consideravam se situar em nível médio; 17 (29,3\%) em baixo e apenas 3 $(5,2 \%)$ em alto. De modo geral, obteve-se nível medianamente estressante, haja vista que o escore total do domínio variou de 0,8 a 6,6 pontos, com média de 4,0 $\pm 1,3$ pontos.

Na Tabela 1 a seguir, consta a distribuição do nível de estresse e resultados da análise univariada do escore médio do Domínio D da EBS, de acordo com variáveis sociodemográficas e profissionais de enfermeiros atuantes em UTI. 
Tabela 1. Distribuição do nível de estresse e resultados da análise univariada do escore médio do Domínio D da EBS, segundo variáveis sociodemográficas e profissionais de enfermeiros intensivistas. Cascavel-PR, 2011.

\begin{tabular}{|c|c|c|c|c|c|c|c|c|c|c|}
\hline \multirow{4}{*}{$\begin{array}{l}\text { VARIÁVEIS } \\
\text { Sexo }\end{array}$} & \multicolumn{6}{|c|}{ NÍVEL DE ESTRESSE - DOMÍNIO D } & \multirow{2}{*}{\multicolumn{4}{|c|}{$\begin{array}{c}\text { ESTATÍSTICA } \\
\text { Análise univariada* }\end{array}$}} \\
\hline & \multicolumn{2}{|c|}{ Baixo } & \multicolumn{2}{|c|}{ Médio } & \multicolumn{2}{|c|}{ Alto } & & & & \\
\hline & $\mathbf{n}$ & $\%$ & $\mathbf{n}$ & $\%$ & $\mathbf{n}$ & $\%$ & \multirow{2}{*}{$\begin{array}{c}\mathbf{F} \\
0,037\end{array}$} & \multirow{2}{*}{$\begin{array}{r}\mathbf{p} \\
0,848\end{array}$} & \multicolumn{2}{|c|}{$\mathrm{IC}_{95 \%}$} \\
\hline & & & & & & & & & $-0,852$ & 0,703 \\
\hline Masculino $(\mathrm{n}=18)$ & 5 & 27,8 & 12 & 66,7 & 1 & 5,6 & & & & \\
\hline Feminino $(n=39)$ & 12 & 30,8 & 25 & 64,1 & 2 & 5,1 & & & & \\
\hline Estado civil & & & & & & & 3,928 & 0,052 & $-1,488$ & 0,008 \\
\hline Com companheiro $(n=40)$ & 9 & 22,5 & 28 & 70,0 & 3 & 7,5 & & & & \\
\hline Sem companheiro $(n=18)$ & 8 & 44,4 & 10 & 55,6 & - & - & & & & \\
\hline Chefe de família & & & & & & & 0,010 & 0,923 & $-0,706$ & 0,778 \\
\hline Sim & 9 & 26,5 & 24 & 70,6 & 1 & 2,9 & & & & \\
\hline Não & 8 & 36,4 & 12 & 54,5 & 2 & 9,1 & & & & \\
\hline Filhos & & & & & & & 2,502 & 0,119 & $-0,091$ & 0,772 \\
\hline Nenhum & 10 & 34,5 & 18 & 62,1 & 1 & 3,4 & & & & \\
\hline 1 & 5 & 25,0 & 13 & 65,0 & 2 & 10,0 & & & & \\
\hline 2 & 2 & 28,6 & 5 & 71,4 & - & - & & & & \\
\hline 4 & - & - & 1 & 100,0 & - & - & & & & \\
\hline Vínculo empregatício & & & & & & & 0,646 & 0,425 & $-0,498$ & 1,166 \\
\hline Único & 12 & 27,3 & 29 & 65,9 & 3 & 6,8 & & & & \\
\hline Múltiplo & 5 & 35,7 & 9 & 64,3 & - & - & & & & \\
\hline Tipo de instituição & & & & & & & 0,162 & 0,689 & $-0,580$ & 0,872 \\
\hline Pública & 6 & 25,0 & 17 & 70,8 & 1 & 4,2 & & & & \\
\hline Privada & 11 & 32,4 & 21 & 61,8 & 2 & 5,9 & & & & \\
\hline Tipo de UTI & & & & & & & 0,059 & 0,809 & $-0,846$ & 0,664 \\
\hline Infantila $^{a}$ & 6 & 28,6 & 13 & 61,9 & 2 & 9,5 & & & & \\
\hline Adulto & 11 & 29,7 & 25 & 67,6 & 1 & 2,7 & & & & \\
\hline Função & & & & & & & 0,719 & 0,400 & $-0,731$ & 1,804 \\
\hline Assistencial & 15 & 28,3 & 35 & 66,0 & 3 & 5,7 & & & & \\
\hline Assistencial e gerencial & 2 & 40,0 & 3 & 60,0 & - & - & & & & \\
\hline Turno & & & & & & & 0,758 & 0,388 & $-1,042$ & 0,411 \\
\hline Diurno & 10 & 31,3 & 22 & 68,8 & - & - & & & & \\
\hline Noturno & 7 & 26,9 & 16 & 61,5 & 3 & 11,5 & & & & \\
\hline Satisfação com renda & & & & & & & 2,212 & 0,143 & $-0,223$ & 1,510 \\
\hline Satisfeito $^{b}$ & 13 & 28,3 & 30 & 65,2 & 3 & 6,5 & & & & \\
\hline Insatisfeito ${ }^{c}$ & 4 & 33,3 & 8 & 66,7 & - & - & & & & \\
\hline 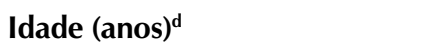 & 28,9 & 4,419 & 31,8 & 4,993 & 37,3 & 7,371 & 7,024 & $\mathbf{0 , 0 1 0} * *$ & 0,021 & 0,152 \\
\hline Tempo em UTI (meses) ${ }^{\mathrm{d}}$ & 29,6 & 9,389 & 41,4 & 27,795 & $33=$ & 077 & 0,966 & 0,330 & $-0,007$ & 0,019 \\
\hline
\end{tabular}

* Teste ANOVA; ${ }^{* *} p \leq 0,05 ;$ Infantil = UTI Pediátrica e UTI Neonatal; ${ }^{b}$ Satisfeito = muito, médio e pouco; ${ }^{c}$ Insatisfeito = muito e pouco; ${ }^{d}$ média \pm DP. 
Tabela 2. Escore médio para cada estressor avaliado no Domínio D por enfermeiros de UTI de cinco instituições hospitalares do Oeste do Paraná. Cascavel-PR, 2010.

\begin{tabular}{|c|c|c|c|c|c|}
\hline \multicolumn{2}{|c|}{ Atividades que compõem a Assistência prestada ao paciente } & \multirow{2}{*}{$\begin{array}{l}\mathrm{n}^{*} \\
58\end{array}$} & \multirow{2}{*}{$\begin{array}{c}\text { Escore } \\
\text { Total }\end{array}$} & \multirow{2}{*}{$\begin{array}{c}\text { Pontuação Média } \\
4,5\end{array}$} & \multirow{2}{*}{$\begin{array}{c}\begin{array}{c}\text { Nível } \\
\text { EBS }\end{array} \\
\text { Médio }\end{array}$} \\
\hline 16 & Admitir o paciente na unidade & & & & \\
\hline 17 & Fazer exame físico do paciente & 56 & 211 & 3,8 & Médio \\
\hline 18 & Prescrever cuidados de enfermagem & 57 & 212 & 3,7 & Médio \\
\hline 19 & Avaliar as condições do paciente & 57 & 209 & 3,7 & Médio \\
\hline 20 & Atender as necessidades do paciente & 58 & 228 & 3,9 & Médio \\
\hline 21 & Atender as necessidades dos familiares & 57 & 251 & 4,4 & Médio \\
\hline 22 & Orientar o paciente para o autocuidado & 42 & 151 & 3,6 & Médio \\
\hline 23 & Orientar os familiares para cuidar do paciente & 51 & 190 & 3,7 & Médio \\
\hline 24 & Supervisionar o cuidado de enfermagem prestado & 58 & 237 & 4,1 & Médio \\
\hline 25 & Orientar para a alta do paciente & 51 & 179 & 3,5 & Médio \\
\hline 26 & Prestar os cuidados de enfermagem & 56 & 216 & 3,9 & Médio \\
\hline 27 & Atender às emergências na unidade & 57 & 288 & 5,1 & Médio \\
\hline 28 & Atender aos familiares de pacientes críticos & 58 & 276 & 4,8 & Médio \\
\hline 29 & Enfrentar a morte do paciente & 58 & 326 & 5,6 & Médio \\
\hline 30 & Orientar familiares de pacientes críticos & 53 & 257 & 4,8 & Médio \\
\hline
\end{tabular}

* Número de respondentes para a respectiva questão, excluindo-se as respostas iguais a zero (0) que se referem às atividades não realizadas pelos sujeitos de pesquisa.

NOTA: As questões estão enumeradas de acordo com a EBS.

Na regressão linear multivariada, a associação entre o escore médio do Domínio D da EBS com a idade perdeu a significância estatística $(p=0,071)$, sendo ajustada pelo estado civil ( $p=0,142)$, quantidade de filhos $(p=0,815)$ e satisfação com a renda $(p=0,176)$.

Ao avaliar o escore médio de cada item/questão, comparando-os entre si, tem-se a possibilidade de identificar a intensidade dos estressores para um enfermeiro ou um grupo particular de enfermeiros ${ }^{(13)}$. Nesse contexto, como consta na Tabela 2, foram verificadas as pontuações médias de cada item que compõe o Domínio $\mathrm{D}$, com vistas ao reconhecimento daquele(s) que mais causam estresse no grupo de enfermeiros.

Apesar de todos os estressores terem sido considerados como sendo de nível mediano, as questões de número 27 , 28, 29 e 30, foram as que obtiveram maior pontuação média.

\section{DISCUSSÃO}

As características sociodemográficas do presente estudo são congruentes aos resultados de outros estudos ${ }^{(4,12,15)}$, haja vista a predominância do sexo feminino $(68,4 \%)$ e a idade média abaixo dos 40 anos. Destarte, o dado da última variável pode ser considerado uma característica inerente ao trabalho desenvolvido em UTI porque, em geral, demandam-se altas cargas físicas compatíveis aos grupos etários mais jovens.
Corrobora a afirmativa anterior o fato de o trabalho em UTI ser marcado pela escassez de tempo, onde tudo é urgente. Nesse contexto, a alta complexidade e a tecnologia avançada implicam na necessidade de profissionais mais especializados e capacitados, sustentando-se um ciclo de rigidez, cobrança e ritmo acelerado ${ }^{(16)}$.

Embora a Assistência prestada ao paciente internado em UTI seja considerada altamente estressante ${ }^{(4,15,17)}$, parece haver uma resposta de adaptação à realização dos itens que compõem este domínio entre os enfermeiros investigados, visto apenas $3(5,2 \%)$ terem apontado alto nível de estresse. Esse resultado se assemelha aos de uma pesquisa recente e pautada no Inventário do Estresse em Enfermeiros ${ }^{(4)}$, a qual detectou que, dentre 21 sujeitos, apenas 5 (23,8\%) consideravam a UTI um local de elevado estresse.

Os dados mencionados anteriormente apontam à necessidade de as lideranças se atentarem à prevenção e/ou manutenção de condições laborais adequadas porque, a assistência ao paciente crítico, apesar de ser desenvolvida em condições estressantes, pode ser percebida como um desafio ou ainda, ser considerada como gratificante ${ }^{(10,17)}$.

É preciso atenção aos aspectos enunciados, que podem impedir que gerentes e gestores percebam e valorizem a seriedade da representação do trabalho do enfermeiro em UTI - o qual se realiza norteado pelo estresse, responsabilidade, assistência 
integral e gratificação - e, por conseguinte, a necessidade de intervenções eficazes para redução do estresse laboral(10).

À análise estatística univariada, apenas a idade se associou ao nível de estresse médio do Domínio D da EBS. Apesar de essa variável perder o poder explicativo à regressão linear multivariada, é importante lembrar que o declínio natural das funções fisiológicas, que ocorre com o envelhecimento, pode resultar em menor capacidade para responder às exigências do trabaIho em UTI e, consequentemente, em maior nível de estresse ocupacional.

Estudo referente ao absenteísmo-doença realizado com profissionais de enfermagem de uma UTI constatou que a idade igual ou superior a 40 anos foi o grupo de profissionais que perde mais dias de trabalho por motivos de doença, quando comparados aos mais jovens ${ }^{(18)}$.

Há carência de pesquisas acerca da prevenção e/ou minimização do estresse no grupo de trabalhadores que se enquadram na faixa etária referida, seja em UTI ou na área da saúde e enfermagem. Contudo, a exemplo de outros campos de atuação - que não se voltam à prestação de cuidados à saúde - pode-se seguir as recomendações de priorização institucional, pela valorização da mão-de-obra madura, com programas de treinamento que contemplem o desenvolvimento de habilidades pró-ativas de enfrentamento do estresse, tais como as habilidades de autorregulação; de análise e resolução de problemas; de tomada de decisões; e competências socioemocionais ${ }^{(19)}$. Associado a isso, em UTI, é preciso ainda considerar aspectos infraestruturais, com adoção de estratégias e dispositivos que diminuam a sobrecarga física e melhorem a ergonomia do trabalho, os quais também pressupõem a penosidade na realização da assistência de enfermagem.

Reconhece-se que o grupo de enfermeiros com 40 anos ou mais é pequeno em UTI, possivelmente porque devido à sua respectiva experiência, estes ocupam cargos administrativos ou atuam em outras áreas que demandam menor capacidade física para o desempenho do trabalho como a de ensino ${ }^{(15)}$; como uma estratégia de fuga ou esquiva pessoal diante à exposição prolongada às condições altamente estressantes em UTI. Destarte, é preciso atenção de gerentes e gestores hospitalares para aqueles que continuam a trabalhar neste setor, visto que, nem sempre, a maturidade possibilita o desenvolvimento de estratégias ativas/pessoais para o enfrentamento do estresse ocupacional ${ }^{(19)}$.

Estudo de revisão de literatura revelou que, tanto o enveIhecimento orgânico natural, quanto o estresse decorrente da organização e do ambiente psicossocial do trabalho têm efeitos importantes sobre a capacidade para o trabalho, em especial para o sexo feminino que além de exercer a dupla jornada de trabalho, apresenta piores condições laborais e de salário, em relação aos homens ${ }^{(20)}$.

Independentemente se consequência ou causa para a menor capacidade para o trabalho, o estresse contribui para o adoecimento dos enfermeiros ${ }^{(21)}$ e com isso, para o aumento do absenteísmo por doença.

Ao se analisar a distribuição do nível de estresse relacionado ao Domínio D da EBS com as demais variáveis sociodemográficas e laborais, chama-se a atenção para o fato de que os
$3(7,5 \%)$ enfermeiros que apresentaram alto nível de estresse possuíam companheiro seja em regime marital ou em matrimonial, trabalhavam em um único emprego, à noite, exclusivamente para prestação de assistência, e sentiam-se satisfeitos com a sua respectiva renda (Tabela 1).

Numa população predominantemente feminina como a deste estudo, o matrimônio ou o convívio marital compreendem, muitas vezes, a responsabilidade pelas tarefas domésticas e educação/criação dos filhos, que ocasionam sobrecarga de atividades, frustração e, consequente nível elevado de estresse. Acresça-se a isso o fato de que a jornada de trabalho em regime de plantão subtrai o tempo livre do enfermeiro e dificulta o convívio social, principalmente no que diz respeito à interação com seus familiares e atividades de lazer, que poderiam ser adotados como estratégias simples para minimizar o estresse ${ }^{(17)}$.

Dada a somatória de estressores vivenciados no trabalho, o enfermeiro pode experimentar conflitos e sentimentos junto a seus familiares que se desdobram em solidão, angústia, incompreensão, irritação e cansaço, dentre outros. Afinal, o cotidiano desse profissional é marcado pelo trabalho solitário e descompasso com o ritmo da família, em que a fadiga limita atividades prazerosas junto aos entes e os afastam pelo descontrole emocional, sob a forma de agressividade, no estabelecimento de suas relações ${ }^{(1)}$.

O trabalho noturno, por sua vez, está associado a níveis de estresse elevados entre profissionais de enfermagem e isso, potencializa a ocorrência de complicações à saúde, uma vez que o desequilíbrio do ritmo biológico, provoca maiores níveis de estresse e pior qualidade do sono ${ }^{(7)}$. Esse fato, provavelmente, influencia a forma como o enfermeiro avalia outros aspectos relacionados à sua atividade laboral, inclusive àqueles que se remetem à Assistência prestada ao paciente.

As condições de trabalho em UTI quando atreladas à atuação noturna, consistem em riscos à saúde dos profissionais de enfermagem. Este dado foi abordado em estudos acerca do estresse e da qualidade do sono entre enfermeiros de diferentes turnos ${ }^{(7,22)}$ os quais constataram que as características dos setores críticos e da atuação noturna geram distúrbios físicos e psíquicos como: alterações hormonais e gástricas causadas pelos ruídos excessivos do setor, pelo ambiente fechado e pela privação do sono.

Dentre os $3(7,5 \%)$ enfermeiros que apontaram alto nível de estresse, no entanto, o trabalho noturno, ao possibilitar incremento salarial com adicionais específicos, pode estar relacionado à satisfação com a renda e consequente manutenção de um único vínculo empregatício. Desse modo, cada uma das atividades que compõem a Assistência prestada ao paciente parece ser o principal motivo que define o nível de estresse dos enfermeiros em UTI.

Conforme consta na Tabela 2, apesar de o nível de estresse ter sido classificado como médio em todos os estressores que compõem a Assistência prestada ao paciente, aqueles que obtiveram pontuação média mais elevada foram: Enfrentar a morte (item 29; 5,6 pontos); Atender às emergências na unidade (item 27; 5,1 pontos); e Atender aos familiares de pacientes críticos ou Orientar familiares de pacientes críticos (itens 28 e 30, respectivamente; ambos com 4,8 pontos). 
No tocante ao nível de estresse laboral devido ao enfrentamento da morte $(5,6$ pontos) e às emergências da unidade (5,1 pontos), vale ressaltar que esses itens foram apontados como os mais estressantes entre os enfermeiros intensivistas, corroborando com outros estudos brasileiros ${ }^{(22-23)}$ que também constaram que o óbito dos pacientes e as situações de emergência são os principais responsáveis pelo desenvolvimento de estresse entre os profissionais de enfermagem.

Ao considerar que a morte de pacientes e a necessidade de atendimentos emergenciais são constantes em UTI, pressupõe-se que haja internalização e aceitação desses eventos como naturais ao processo de trabalho ali realizado. Isso pode ser explicado pela alta sofisticação tecnológica existente neste setor, contexto em que se prevalece, muitas vezes, um trabalho extremamente tecnicista, aliado ao fato de que o ser humano, de modo geral, não é preparado para conviver e/ou aceitar qualquer tipo de perda.

A premissa anterior se remete às formas de adaptação e defesa ao estresse decorrente de situações críticas como a morte e atendimentos emergenciais, que podem resultar em sofrimento psíquico e desgaste emocional nos profissionais de enfermagem que atuam em UTI, pois mediante a proximidade e o envolvimento mantidos durante o período de internação, com o paciente e seus familiares, o óbito daquele tende a exacerbar os sentimentos de impotência, frustração e até mesmo de projeção do sofrimento no enfermeiro ${ }^{(11,17,24)}$.

No que tange às situações emergenciais, o estresse, provavelmente, advém da luta contra o tempo - afinal, a tomada de decisão rápida e precisa associada à disponibilidade de recursos e agilidade da equipe pressupõe a diferença entre a vida e a morte das pessoas. Um estudo descritivo realizado em uma UTI de um hospital universitário brasileiro ${ }^{(25)}$, constatou que a prevalência de estresse ocupacional entre profissionais de enfermagem que mencionaram como causas, a gravidade dos pacientes e a instabilidade do quadro clínico, era de 59,4\%.

Orientar e/ou atender os familiares de pacientes críticos também não é tarefa fácil haja vista que, independentemente do nível de escolaridade ou do grau de compreensão, a família vivencia um momento ímpar em que sentimentos relacionados ao adoecimento de um ente querido, associada à negação da gravidade da doença, à ansiedade e o medo da morte, podem representar empecilho ao entendimento e à interação com a equipe da UTI e, por conseguinte, causar estresse ocupacional. O momento da orientação e atendimento aos familiares, portanto, podem ser considerados como estressantes pelos enfermeiros que devido à aproximação com a família do paciente, desencadeia um processo de identificação de sentimentos, com envolvimento para além do paciente ${ }^{(24)}$.

É importante lembrar que, como dito anteriormente, o estresse se relaciona à percepção e compreensão individual da situação que cada qual vivencia. Com isso, o profissional, ao identificar quais são os fatores que o estressa, de alguma forma, precisa desenvolver estratégias para lidar com o que lhe incomoda e assim, melhorar a sua qualidade de vida e de trabalho(4).

Apesar de presente em toda e qualquer situação, além de ser uma atividade corriqueira do ser humano, cada sujeito tem a possibilidade de reagir de forma diferenciada aos diversos estímulos aos quais estão expostos. Desse modo, a forma como o indivíduo reage determina o nível de estresse que ele foi submetido e que mudanças serão implementadas para superá-lo(6).

É preciso, entretanto, que as estratégias individuais e em grupo sejam implantadas e/ou intermediadas por gerentes e gestores porque, o estresse profissional tende a afetar a vida pessoal e também, o desempenho profissional dos enfermeiros.

\section{CONCLUSÕES}

Os enfermeiros investigados, que atuam em UTI, são predominantemente do sexo feminino $(68,4 \%)$, com média de idade abaixo dos 40 anos $(91,4 \%)$.

Apenas 3 (5,2\%) enfermeiros apontaram que a Assistência prestada ao paciente redunda em alto nível de estresse. Esse dado indica que essa categoria profissional se adapta ao ambiente de trabalho reconhecido pela literatura como altamente estressante.

O modelo de regressão linear multivariada não apresentou associação significativa entre variáveis sociodemográficas e profissionais com o nível médio de estresse do Domínio D da EBS.

Todos os estressores que compõem o domínio Assistência prestada ao paciente foram considerados como medianamente estressantes. Especial atenção deve ser dada, entretanto, àqueles que obtiveram pontuações mais elevadas e que representam potenciais agravantes à saúde dos enfermeiros, dentre os quais constam: Enfrentar a morte (5,6 pontos); Atender às emergências na unidade (5,1 pontos); e, Atender aos familiares de pacientes críticos ou Orientar familiares de pacientes críticos (respectivamente com 4,8 pontos).

Os dados deste estudo indicam que o estresse entre os enfermeiros das UTI investigadas não é elevado, mas se encontra presente nos diferentes momentos em que presta assistência ao paciente. Para minimizar esse quadro, sugere-se que as instituições e seus trabalhadores adotem estratégias voltadas à sua prevenção e/ou redução, sem que os profissionais deixem de exercer as atividades previstas em Lei.

\section{REFERÊNCIAS}

1. Fernandes SMBA, Medeiros SM, Ribeiro LM. Estresse ocupacional e o mundo do trabalho atual: repercussões na vida cotidiana das enfermeiras. Rev Eletr Enferm. 2008;10(2):414-27. [Acesso em 20 jul. 2011]. Disponível em: http://www.fen.ufg.br/revista/v10/n2/v10n2a13.htm
2. Azevedo VAZ, Kitamura S. Stress, Trabalho e Qualidade de Vida. In: Vilarta R, Org. Qualidade de vida e fadiga institucional. Campinas: IPES Editorial; 2006. p. 137-150.

3. Houtmann I, Jettinghoff K, Cedillo L, Orgs. Raising awareness of stress at work in developing countries: a modern 
hazard in a traditional working environment: advice to employers and worker representatives. Protecting worker's health, n. 6. Geneva: World Health Organization; 2007.

4. Preto VA, Pedrao LJ. O estresse entre enfermeiros que atuam em Unidade de Terapia Intensiva. Rev Esc Enferm USP. 2009;43(4):841-8.

5. Griep RH, Rotenberg L, Landsbergis P, Vasconcellos-Silva PR. Uso combinado de modelos de estresse no trabalho e a saúde auto-referida na enfermagem. Rev Saúde Pública. 2011;45(1):145-52.

6. Hanzelmann RS, Passos JP. Imagens e representações da enfermagem acerca do stress e sua influência na atividade laboral. Rev Esc Enferm USP. 2010;44(3):694-701.

7. Rocha MCP, Martino MMF. O estresse e qualidade de sono do enfermeiro nos diferentes turnos hospitalares. Rev Esc Enferm USP. 2010;44(2):280-6.

8. Wu H, Chi TS, Chen L, Wang L, Jin YP. Occupational stress among hospital nurses: cross-sectional survey. J Adv Nurs. 2010;66(3):627-34.

9. Li J; Lambert VA. Workplace stressors, coping, demographics and job satisfaction in Chinese intensive care nurses. Nurs Crit Care. 2008;13(1):12-24.

10. Silva IAS, Cruz EA. Trabalho da enfermeira intensivista: um estudo da estrutura das representações sociais. Rev Esc Enferm USP. 2008;42(3):554-62.

11. Shorter M, Stayt LC. Critical care nurses' experiences of grief in an adult intensive care unit. J Adv Nurs. 2010;66(1):159-67.

12. Cavalheiro AM, Moura Junior DF, Lopes AC. Stress in nurses working in intensive care units. Rev Latino-Am Enfermagem. 2008;16(1):29-35.

13. Bianchi ERF. Escala Bianchi de Stress. Rev Esc Enferm USP. 2009;43(spe):1055-62.

14. Brasil. Decreto-Lei n 94406 de 8 de junho de 1987. Brasília (DF): Ministério da Saúde; 1987.

15. Guerrer FJL, Bianchi ERF. Caracterização do estresse nos enfermeiros de unidades de terapia intensiva. Rev Esc Enferm USP. 2008;42(2):355-62.
16. Campos JF, David HSL. Avaliação do contexto de trabalho em terapia intensiva sob o olhar da psicodinâmica do trabalho. Rev. Esc. enferm. USP. 2011;45(2):363-8.

17. Santos F D, Cunha MHF, Robazzi CC et al. O estresse do enfermeiro nas unidades de terapia intensiva adulto: uma revisão da literatura. SMAD, Rev. Eletrônica Saúde Mental Álcool Drog. (Ed. port.) [Internet]. 2010[cited 2011 jan 11]; 6(1):1-16. Available from: http://pepsic.bvsalud.org/scielo. php?script $=$ sci_arttext\&pid $=$ S1806-69762010000100014\&ln $\mathrm{g}=\mathrm{pt \& nrm}=$ iso $>$. ISSN 1806-6976.

18. Inoue KC, Matsuda LM, Silva DMPP, Uchimura TT, Mathias TAF. Absenteísmo-doença da equipe de enfermagem em unidade de terapia intensiva. Rev Bras Enferm 2008;61(2):209-14.

19. Fontes AP, Neri AL, Yassuda MS. Enfrentamento de estresse no trabalho: relações entre idade, experiência, autoeficácia e agência. Psicol. cienc. prof. 2010;30(3):620-33.

20. Martinez MC, Latorre MRDO, Fischer FM. Capacidade para o trabalho: revisão de literatura. Ciênc. saúde coletiva. 2010;15(suppl.1):1553-61.

21. Frutuoso JT, Cruz RM. Mensuração da carga de trabalho e sua relação com a saúde do trabalhador. Rev Bras Med Trab. 2005;3(1):29-36.

22. Guido LA, Umann J, Stekel LMC et al. Estresse, coping e estado de saúde de enfermeiros de clínica médica em um hospital universitário. Cienc Cuid Saúde. 2009; 8(4):615-621.

23. Rodrigues $A B$, Chaves EC. Fatores estressantes e estratégias de coping dos enfermeiros atuantes em oncologia. Rev Latino-Am Enfermagem. 2008;16(1):24-8.

24. Martins JT, Robazzi MLCC. Nurses' work in intensive care units: feelings of suffering. Rev Latino-Am Enfermagem. 2009;17(1):52-8.

25. Miranda EJP, Stancato K. Riscos à saúde de equipe de enfermagem em unidade de terapia intensiva: proposta de abordagem integral da saúde. Rev Bras Ter Intensiva [Internet]. 2008 [cited 2011 jan 10];20(1):68-76. Available from: http://www.scielo.br/scielo.php?script $=$ sci arttext\&pid $=$ S0103-507X2008000100011\&Ing $=$ en . doi: 10.1590/S0103-507X2008000100011. 